Commun. Korean Math. Soc. 18 (2003), No. 2, pp. 289-295

\title{
ON RULED SURFACES IN MINKOWSKI SPACES
}

\author{
Dong-Soo Kim, Young Ho Kim AND DaE Won YoON
}

\begin{abstract}
In this paper, we study ruled surfaces in Minkowski space, which admit 1-type Gauss map. In particular, non-cylindric -al ruled surfaces with finite type Gauss map are of $k$-type $(k \geq 2)$.
\end{abstract}

\section{Introduction}

Submanifolds of finite type in Euclidean or pseudo-Euclidean space were introduced in late 1970's ([5]). Since then, many works have been achieved in this regard. Moreover, the notion of finite type can be extended to the smooth maps on submanifolds such as the Gauss map. By definition, the Gauss map $G$ on a submanifold $M$ in a pseudo-Euclidean space $\mathbb{E}_{s}^{m}$ with signature $(s, m-s)$ is of finite type if $G$ can be expressed as a finite sum of eigenvectors of the Laplacian $\Delta$ of $M$, that is,

$$
G=G_{0}+G_{1}+G_{2}+\cdots+G_{k}
$$

where $G_{0}$ is a constant map, $G_{1}, \ldots, G_{k}$ non-constant maps such that $\Delta G_{i}=\lambda_{i} G_{i}, \lambda_{i} \in \mathbb{R}, i=1,2, \ldots, k$. If $\lambda_{1}, \lambda_{2}, \ldots, \lambda_{k}$ are different, then $G$ is said to be of $k$ - type. In particular, if one of $\lambda_{1}, \lambda_{2}, \ldots, \lambda_{k}$ is zero, then it is called the null $k$-type.

The authors studied ruled surfaces over non-null base curve in Minkow -ski space with finite type Gauss map $([8,9,10])$ and classified null scrolls with 1-type Gauss map which are called the extended $B$-scrolls ([10]).

In this article, we improve some results on ruled surfaces in an $m$ dimensional Minkowski space $\mathbb{E}_{1}^{m}$ with finite type Gauss map. Throughout this paper, we assume that all objects are smooth and all surfaces are connected unless stated otherwise.

Received April 12, 2002.

2000 Mathematics Subject Classification: 53B25, 53C50.

Key words and phrases: Minkowski space, Gauss map, finite type, ruled surface. 


\section{Preliminaries}

Let $\mathbb{E}_{s}^{m}$ be an $m$-dimensional pseudo-Euclidean space with signature $(s, m-s)$. Then the metric tensor $\tilde{g}$ in $\mathbb{E}_{s}^{m}$ has the form

$$
\tilde{g}=-\sum_{i=1}^{s} d x_{i}^{2}+\sum_{i=s+1}^{m} d x_{i}^{2},
$$

where $\left(x_{1}, x_{2}, \ldots, x_{m}\right)$ is a standard rectangular coordinate system in $\mathbb{E}_{s}^{m}$. In particular, for $m \geq 2, \mathbb{E}_{1}^{m}$ is called a Minkowski m-space.

Let $x: M \longrightarrow \mathbb{E}_{s}^{m}$ be a isometric immersion of an oriented $n$ dimensional pseudo-Riemannian submanifold $M$ into $\mathbb{E}_{s}^{m}$.

From now on, a submanifold in $\mathbb{E}_{s}^{m}$ always means pseudo-Riemannian unless stated otherwise.

Let $G(n, m)$ be the Grassmannian manifold consisting of all oriented $n$-planes through the origin of $\mathbb{E}_{s}^{m}$ and $\bigwedge^{n} \mathbb{E}_{s}^{m}$ the vector space determined by the exterior product of $n$ vectors in $\mathbb{E}_{s}^{m}$. Let $e_{1}, e_{2}, \ldots, e_{m}$ be an adapted local orthogonal frame in $\mathbb{E}_{s}^{m}$ such that $e_{1}, e_{2}, \ldots, e_{n}$ are tangent to $M$ and $e_{n+1}, e_{n+2}, \ldots, \dot{e}_{m}$ normal to $M$. The map $G: M \longrightarrow$ $G(n, m)$ defined by $G(p)=\left(e_{1} \wedge e_{2} \wedge \cdots \wedge e_{n}\right)(p)$ is called the Gauss map of $M$ that is a smooth map which carries a point $p$ in $M$ into the oriented $n$-plane in $\mathbb{E}_{s}^{m}$ obtained from the parallel translation of the tangent space of $M$ at $p$ in $\mathbb{E}_{s}^{m}$.

For two vectors $e_{i_{1}} \wedge \cdots \wedge e_{i_{n}}$ and $f_{j_{1}} \wedge \cdots \wedge f_{j_{n}}$ of $\mathbb{E}_{k}^{N}$, we can define an indefinite inner product $\langle$,$\rangle by$

$$
\left\langle e_{i_{1}} \wedge \cdots \wedge e_{i_{m}}, f_{j_{1}} \wedge \cdots \wedge f_{j_{m}}\right\rangle=\operatorname{det}\left(\left\langle e_{i_{l}}, f_{j_{k}}\right\rangle\right)
$$

on $G(n, m)$. Then, $\wedge^{n} \mathbb{E}_{s}^{m}$ can be viewed as a pseudo-Euclidean space $\mathbb{E}_{k}^{N}$ for some positive integer $k$ where $N=\left(\begin{array}{c}m \\ n\end{array}\right)$ and $G(n, m)$ lies in a unit pseudo-hypersphere $S_{k}^{N-1}(1) \subset \mathbb{E}_{k}^{N}$.

Let $\left\{x_{1}, x_{2}, \cdots, x_{n}\right\}$ be a local coordinate system of $M$. For the components $g_{i j}$ of the pseudo-Riemannian metric $\langle$,$\rangle on M$ we denote by $\left(g^{i j}\right)$ (resp. $\mathcal{G}$ ) the inverse matrix (resp. the determinant) of the matrix $\left(g_{i j}\right)$. As is well known, the Laplacian $\Delta$ on $M$ is given by

$$
\Delta=-\frac{1}{\sqrt{|\mathcal{G}|}} \sum_{i, j} \frac{\partial}{\partial x^{i}}\left(\sqrt{|\mathcal{G}|} g^{i j} \frac{\partial}{\partial x^{j}}\right) .
$$

Now, we define a ruled surface $M$ in $\mathbb{E}_{1}^{m}$. Let $I$ and $J$ be open intervals containing 0 in the real line $\mathbb{R}$. Let $\alpha=\alpha(s)$ be a curve on in $\mathbb{E}_{1}^{m}$ defined 
on $J$ and $\beta=\beta(s)$ a transversal vector field along $\alpha$. So we have the parametrization for $M$

$$
x=x(s, t)=\alpha(s)+t \beta(s), \quad s \in J, \quad t \in I .
$$

We call $\alpha$ the base curve and $\beta$ the director curve. In particular, if $\beta$ is constant, the ruled surface is said to be cylindrical, and if it is not so, it is called non-cylindrical. In this paper, we consider that the base curve $\alpha$ is space-like or time-like. In the case, the director curve $\beta$ can be naturally chosen so that it is orthogonal to $\alpha$. Furthermore, we have ruled surfaces of five different kinds according to the character of the base curve $\alpha$ and the director curve $\beta$ as follows: If the base curve $\alpha$ is space-like or time-like, then the ruled surface $M$ is said to be of type $M_{+}$or type $M_{-}$, respectively. Also, the ruled surface of type $M_{+}$can be divided into three types. In the case that $\beta$ is space-like, it is said to be of type $M_{+}^{1}$ or $M_{+}^{2}$ if $\beta^{\prime}$ is non-null or null, respectively. When $\beta$ is time-like, $\beta^{\prime}$ must be space-like according to the causal character. In this case, $M$ said to be of type $M_{+}^{3}$. On the other hand, for the ruled surface of type $M_{-}$, it is also said to be of type $M_{-}^{1}$ or $M_{-}^{2}$ if $\beta^{\prime}$ is non-null or null, respectively. Note that in the case of type $M_{-}$the director curve $\beta$ is always space-like. The ruled surface of type $M_{+}^{1}$ or $M_{+}^{2}$ (resp. $M_{+}^{3}, M_{-}^{1}$ or $M_{-}^{2}$ ) is clearly space-like (resp. time-like)([8]).

\section{Main results}

Let the Gauss map $G$ of the ruled surface $M$ in $\mathbb{E}_{1}^{m}$ is of 1-type. Then, as is given [4] and [5], there exists a real number $\lambda$ such that

$$
\Delta^{2} G+\lambda \Delta G=0
$$

TheOREM 3.1. There is no non-cylindrical ruled surfaces over a nonnull base curve with 1-type Gauss map.

Proof. Let $M$ be a non-cylindrical ruled surface in $\mathbb{E}_{1}^{m}$ of one of three types $M_{+}^{1}, M_{+}^{3}$ or $M_{-}^{1}$. According to Theorem 3.2 in [8], it is impossible for $G$ to be of 1-type.

Let $M$ be a non-cylindrical ruled surface of type $M_{+}^{2}$ or $M_{-}^{2}$. The parametrization for $M$ is given by

$$
x(s, t)=\alpha(s)+t \beta(s)
$$


such that $\langle\beta, \beta\rangle=1, \quad\left\langle\alpha^{\prime}, \beta\right\rangle=0, \quad\left\langle\alpha^{\prime}, \alpha^{\prime}\right\rangle=\varepsilon_{1}(= \pm 1)$ and $\beta^{\prime}$ is null. It is easy to get the Gauss map of the surface $M$ as

$$
G=\frac{1}{\left\|\left(\alpha^{\prime}+t \beta^{\prime}\right) \wedge \beta\right\|}\left(\alpha^{\prime}+t \beta^{\prime}\right) \wedge \beta .
$$

Define functions $q$ and $u$ by

$$
q=\left\|x_{s}\right\|^{2}=\varepsilon_{4}\left\langle x_{s}, x_{s}\right\rangle, \quad u=\left\langle\alpha^{\prime}, \beta^{\prime}\right\rangle,
$$

which give

$$
q=\varepsilon_{4}\left(2 u t+\varepsilon_{1}\right), \quad G=\frac{1}{q^{\frac{3}{2}}}(A+t B)
$$

where we put $A=\alpha^{\prime} \wedge \beta$ and $B=\beta^{\prime} \wedge \beta$ and the region of $t$ runs so that $q>0$. By the straightforward computation, we easily have the Laplacian $\Delta$ of $M$ in the form

$$
\Delta=-\varepsilon_{4}\left(-\frac{1}{2} \frac{1}{q^{2}} \frac{\partial q}{\partial s} \frac{\partial}{\partial s}+\frac{1}{q} \frac{\partial^{2}}{\partial s^{2}}\right)-\left(\frac{1}{2} \frac{1}{q} \frac{\partial q}{\partial t} \frac{\partial}{\partial t}+\frac{\partial^{2}}{\partial t^{2}}\right)
$$

On the other hand, we can obtain the following lemma.

Lemma. If $P$ is a polynomial in $t$ with functions in $s$ as coefficients and $\operatorname{deg}(P)=d$, then $\Delta\left(\frac{P(t)}{q^{m}}\right)=\frac{1}{q^{m+3}} \tilde{P}(t)$ where $\tilde{P}(t)$ is a polynomial in $t$ with functions in $s$ as coefficients and $\operatorname{deg}(\tilde{P}) \leq d+2$.

For the Gauss map $G$, we have by Lemma

$$
\Delta G=\frac{G_{1}(t)}{q^{\frac{1}{2}+3}}, \ldots, \Delta^{r} G=\frac{G_{r}(t)}{q^{\frac{1}{2}+3 r}}, \quad \operatorname{deg}\left(G_{r}(t)\right) \leq 1+2 r
$$

where $G_{1}(t), \cdots, G_{r}(t)$ are polynomials in $t$ as coefficients.

We now consider an open subset $\mathcal{U}=\left\{p \in M \mid\left(u^{2}\right)^{\prime}(p) \neq 0\right\}$. Suppose that $\mathcal{U}$ is not empty. Then, the above lemma gives $\Delta G=0$ by the argument of degree of polynomials in $t$ on $\mathcal{U}$.

Using (3.2) and (3.3), we can obtain by a direct computation

$$
\begin{aligned}
\Delta G= & \left(-2 u^{2} q^{-2}+u^{\prime \prime} t q^{-2}-4 \varepsilon_{4} u^{\prime 2} t^{2} q^{-3}\right) G \\
& +q^{-\frac{5}{2}}\left\{\varepsilon_{4} u B q+3 u^{\prime} t\left(A^{\prime}+t B^{\prime}\right)-\varepsilon_{4}\left(A^{\prime \prime}+t B^{\prime \prime}\right) q\right\}
\end{aligned}
$$


By using (3.4) and (3.5) we have the following equations:

$$
\begin{gathered}
2 u^{2} B^{\prime \prime}-3 u u^{\prime} B^{\prime}+2 u^{2} B-u u^{\prime \prime} B=0, \\
4 u^{2} A^{\prime \prime}-6 u u^{\prime} A^{\prime}-2 u u^{\prime \prime} A+4 u^{\prime 2} A \\
+4 \varepsilon_{1} u B^{\prime \prime}-3 \varepsilon_{1} u^{\prime} B^{\prime}+\varepsilon_{1} u^{\prime \prime} B=0, \\
4 \varepsilon_{1} u A^{\prime \prime}-3 \varepsilon_{1} u^{\prime} A^{\prime}+4 u^{3} A-\varepsilon_{1} u^{\prime \prime} A+B^{\prime \prime}-2 \varepsilon_{1} u^{2} B=0, \\
A^{\prime \prime}+2 \varepsilon_{1} u^{2} A-u B=0 .
\end{gathered}
$$

From (3.5)-(3.9), we can eliminate $A^{\prime \prime}, B^{\prime \prime}, A^{\prime}$ and $B^{\prime}$ so that

$$
2 \varepsilon_{1} u u^{\prime 2} A=\left(u^{\prime 2}-u u^{\prime \prime}\right) B,
$$

from which, the basic properties of $\beta$ give $\alpha^{\prime}=\rho \beta^{\prime}$ for some function $\rho$ on $\mathcal{U}$. It is a contradiction. Thus, the open subset $\mathcal{U}$ is empty, that is, $u$ is constant. Suppose $u \neq 0$. (3.6)-(3.9) imply that $u A-1 / 2 \epsilon_{1} B=0$, which gives $u \alpha^{\prime}=1 / 2 \epsilon_{1} \beta^{\prime}$, a contradiction. Thus, $u$ is identically zero on $M$.

Let $M$ be a surface of type $M_{-}^{2}$. It is impossible because there is no time-like vector orthogonal to a null vector in Minkowski space.

If $M$ is a surface of type $M_{+}^{2}$, then it is easily seen that $M$ is flat space-like. Since the Gauss map $G$ is of 1-type and the function $u=0$, we have

$$
\Delta G+\lambda G+\tilde{C}=0
$$

in other words,

$$
-\left(A^{\prime \prime}+t B^{\prime \prime}\right)=\lambda(A+t B)+\tilde{C}
$$

for some constant vector $\tilde{C}$, from which,

$$
A^{\prime \prime}=-\lambda A-\tilde{C}, \quad \tilde{C}=-\lambda B .
$$

On the other hand, since $\beta^{\prime}$ is a null vector field, $B$ is a null vector field. So, $B^{\prime \prime}=-\lambda B$ implies $\left\langle\beta^{\prime \prime}, \beta^{\prime \prime}\right\rangle=0$.

Suppose $\beta^{\prime \prime}$ is a null vector field. By the causal character of $\beta^{\prime}$, $\beta^{\prime \prime}=\tilde{\rho} \beta^{\prime}$ for some function $\tilde{\rho}$. Therefore, $\beta=F(s) \mathbb{N}$ for some null 
constant vector $\mathbb{N}$, which is a contradiction. Hence, the vector field $\beta^{\prime \prime}$ is identically zero on $M$. Thus, the vector field $\beta$ is given by

$$
\beta(s)=s \mathbb{D}+\mathbb{E}
$$

for some null constant vector $\mathbb{D}$ and a unit constant space-like vector $\mathbb{E}$ such that $\langle\mathbb{D}, \mathbb{E}\rangle=0$.

From the first equation of (3.11), we get

$$
\begin{aligned}
\left(s \alpha^{\prime \prime \prime \prime}(s)\right. & \left.+3 \alpha^{\prime \prime \prime}(s)+\lambda s \alpha^{\prime \prime}(s)+\lambda \alpha^{\prime}(s)\right) \wedge \mathbb{D} \\
& +\left(\alpha^{\prime \prime \prime \prime}(s)+\lambda \alpha^{\prime \prime}(s)\right) \wedge \mathbb{E}=0,
\end{aligned}
$$

from which, $\lambda=0$ and thus $M_{+}^{2}$ must be of null 1-type, in other words, $\Delta G=0$ is satisfied. In turn, the base curve $\alpha$ is a straight line with $\alpha(s)=s \mathbb{F}$ for some constant space-like unit vector $\mathbb{F}$ up to congruence. Since $\left\langle\alpha^{\prime}, \beta\right\rangle=0, \mathbb{F}$ is orthogonal to $\mathbb{D}$ and $\mathbb{E}$ respectively. Consequently, $M_{+}^{2}$ can be parametrized by $x(s, t)=(s t, s t, t, s)$ in $\mathbb{E}_{1}^{4}$ which is isometric to a Euclidean plane which is cylindrical, a contradiction.

Therefore, by combining the result of [9] and above, we have

COROLLARY 3.2. Let $M$ be a non-cylindrical ruled surface over nonnull base curve in $\mathbb{E}_{1}^{m}$ with $k$-type Gauss map. Then, it is of type $M_{+}^{2}$ and $k \geq 2$.

REMARK.([10]) There are abundant examples of flat non-cylindrical $M_{+}^{2}$-type ruled surfaces in $\mathbb{E}_{1}^{m}(m \geq 4)$ with $k$-type Gauss map $(k \geq 2)$.

EXAMPLE. Let $\alpha$ be a space-like curve of the form $\alpha=\alpha(s)=$ $\left(c e^{s}, c e^{s}, 0, s\right)$ and $\beta$ a vector field along $\alpha$ such that $\beta=\beta(s)=(s, s, 1,0)$ in $\mathbb{E}_{1}^{4}$ where $c(\neq 0)$ is a real number. Consider a ruled surface $M$ parametrized by $x(s, t)=\alpha(s)+t \beta(s)$ on $s \in I$ and $t \in J$ for some open intervals $I$ and $J$. Then, $M$ is a non-cylindrical ruled surface of type $M_{+}^{2}$ and the Gauss map $G$ of $M$ satisfies $\Delta^{2} G+\Delta G=0, \Delta G \neq 0$.

Putting together Theorem 3.1 and Theorem in $[8,9,10]$, we get

THEOREM 3.3. Let $M$ be a ruled surface in $\mathbb{E}_{1}^{m}$ with 1-type Gauss map. Then, $M$ is either cylindrical over 1-type non-null base curve or an extended $B$-scroll. 


\section{References}

[1] C. Baikoussis and D. E. Blair, On the Gauss map of ruled surfaces, Glasgow Math. J. 34 (1992), 355-359.

[2] C. Baikoussis, B.-Y. Chen and L. Verstraelen, Ruled surfaces and tubes with finite type Gauss map, Tokyo J. Math. 16 (1993), 341-348.

[3] - Surfaces with finite type Gauss map, Geometry and Topology of Submanifolds IV World Scientific Publ. (1992), 214-216.

[4] B.-Y. Chen, On submanifolds of finite type, Soochow J. Math. 9 (1983), 65-81.

[5] , Total Mean Curvature and Submanifolds of Finite Type, World Scientific Publ., 1984.

[6] B.-Y. Chen and M. Petrovic, On spectral decomposition of immersions of finite type, Bull. Austral. Math. Soc. 44 (1991), 117-129.

[7] B.-Y. Chen and P. Piccinni, Submanifolds with finite type Gauss map, Bull. Austral. Math. Soc. 35 (1987), 161-186.

[8] Y. H. Kim and D. W. Yoon, Ruled surfaces with finite type Gauss map in Minkowski spaces, Soochow J. Math. 26 (2000), 85-96.

[9] - Correction to:Ruled surfaces with finite type Gauss map in Minkowski spaces, to appear in Soochow J. Math.

[10] D.-S. Kim, Y. H. Kim and D. W. Yoon, Extended B-scrolls and their Gauss maps, to appear in Ind. J. Pure and Appl. Math..

Dong-Soo Kim

Department of Mathematics

Chonnam National University

Kwangju 500-757, Korea

E-mail: dosokim@chonnam.ac.kr

Young Ho Kim

Department of Mathematics

Teachers College

Kyungpook National University

Taegu 702-701, Korea

E-mail: yhkim@kyungpook.ac.kr

Dae Won Yoon

Department of Mathematics

College of Education

Gyeongsang National University

Chinju 660-701, Korea

E-mail: dwyoon@gauss.knu.ac.kr 